\title{
Effects of gelatin combined with essential oils coating on storage quality of turbot (Psetta maxima) fillets
}

\author{
Yongxia $\mathrm{Xu}^{1,2}$, Rui Wang ${ }^{2}$, Honglei Zhao ${ }^{2}$, Jiamei Zhao ${ }^{2}$, Jianping Zhang ${ }^{1, *}$, Jianrong $\mathrm{Li}^{2,}$, \\ ${ }^{1}$ Jiangsu Key Lab of Food Resource Development and Quality Safety, Xuzhou Institute of \\ Technology, Xuzhou 221111, China \\ ${ }^{2}$ College of Food Science and Technology, Bohai University, Jinzhou 121013, China \\ E-mail: xuyx1009@126.com
}

Keywords: Turbot; Garlic essential oil; Ginger essential oil; Coating preservation

\begin{abstract}
The influences of garlic and ginger essential oil coating on the quality of turbot (Psetta maxima) fillets during storage were investigated. Total viable count (TVC), total volatile basic nitrogen (TVB-N), $\mathrm{pH}$ and texture profile were measured to evaluate the preservative effect of essential oil coating on turbot fillets. The results suggested that the treatment group revealed an obvious preservative effect, and the best was garlic essential oil coating, resulting in an extension of shelf-life by 4 days. Meanwhile, changes of TVC, TVB-N, pH and texture profile of turbot fillets were significantly reduced by essential oil treatment compared to the control group. The essential oil coating showed a significant inhibitory effect on the bacteria propagation and slowed down the protein degradation, thus prolonged the shelf-life of turbot fillets during refrigeration.
\end{abstract}

\section{Introduction}

Turbot, as a mercantile marine fish species, is popular in the market due to its white and flavourful flesh, abundant pectin and high nutritional quality[1]. However, fragile muscular tissue and active cathepsin lead to the short period of rigor stage and the faster speed of autolysis and corruption in fish during the storage. Nowadays, with the development of organic food, the development and application of bio-preservatives has become the hot spot and trend in the aquatic products preservation. Spices, such as ginger, garlic, cinnamomum cassia and clove, have been widely used in fish preservation due to its effective antioxidation and antimicrobial activities [2]. It has been reported that the main active constituent of ginger oil were 6-gingerol that can reduce formation of pseudomonas aeruginosa biofilm, restrain quorum sensing (QS) inducible gene expression and lipid oxidation during the refrigerated storage[3]. Additionally, Teixeira et al. [4] investigated the antioxidant and antibacterial properties of fish protein films incorporated with essential oils of clove, garlic and origanum, and found that garlic films presented the highest antioxidant activity. However, to our knowledge, little information is available on the response of turbot fillets to essential oil treatment. Thus, in this paper, the effect of ginger and garlic essential oil coating on the quality of turbot fillets during storage at $4^{\circ} \mathrm{C}$ for 20 days was evaluated.

\section{Materials and methods}

\subsection{Preparation of sample and treatment}

Fresh turbot with an average weight of 1.0-1.2 kg were aquired from a local seafood market (Jinzhou, China) and carried to the Aquatic Products Processing Lab of Bohai University within $1 \mathrm{~h}$. Fish were killed and filleted by hand. The gelatin coating solution (6\%), including sorbitol (1\%) and glycerol (1\%), which were added as plasticizers. Food grade essential oil (2\%) was incorporated, 
and tween-80 (2\%) was added as an emulsifying agent. Three different types of coatings were then obtained: a gelatin coating; a gelatin ginger-added coating and a gelatin garlic-added coating. The fillets were immediately soaked in coating solution for $10 \mathrm{~min}$. After draining, all samples were packed by aseptic polyethylene bags and stored at $4 \pm 1{ }^{\circ} \mathrm{C}$ during 20 days. Fish samples were taken subsequently every 4 days for quality evaluation.

\subsection{Microbiological analysis}

TVC was performed using aerobic plate counts (APCs) according to the method of Chinese National Standard (GB/T 4789.2-2016).

\subsection{TVB-N value}

TVB-N content was determined by the FOSS method [5]. TVB-N values were measured with a Foss Kjeltec 8400 (FOSS, Sweden) and expressed in mg N per $100 \mathrm{~g}$ fish muscle.

\section{4. $\mathrm{pH}$}

Minced turbot samples $(10 \mathrm{~g})$ were homogenised with $90 \mathrm{ml}$ boiled distilled water. The mixture was filtered and stewed for $30 \mathrm{~min}$, and then the $\mathrm{pH}$ of supernatant was measured with a $\mathrm{pH}$ meter (Mettler Toledo, Zurich, Switzerland).

\subsection{Texture analysis}

Texture profile analyses (TPA) were carried out using a TA-XT2i Texture Analyzer (Stable Micro Systems $\left.{ }^{\circledR}\right)$. The dorsal muscle of fillets $(1.5 \times 1.5 \times 1.0 \mathrm{~cm})$ was compressed perpendicularly twice to $75 \%$ of its original height. The speed of probe was $1 \mathrm{~mm} / \mathrm{s}$ during penetration.

\subsection{Statistical analysis}

Each sample was repeated at least in triplicate. The data charts were made with the program of Excel and date analysis were measured with SPASS statistical program (Version 19.0), differences at $p<0.05$ were defined significant.

\section{Results and discussion}

\subsection{Effects of essential oils coating on TVC of turbot fillets}

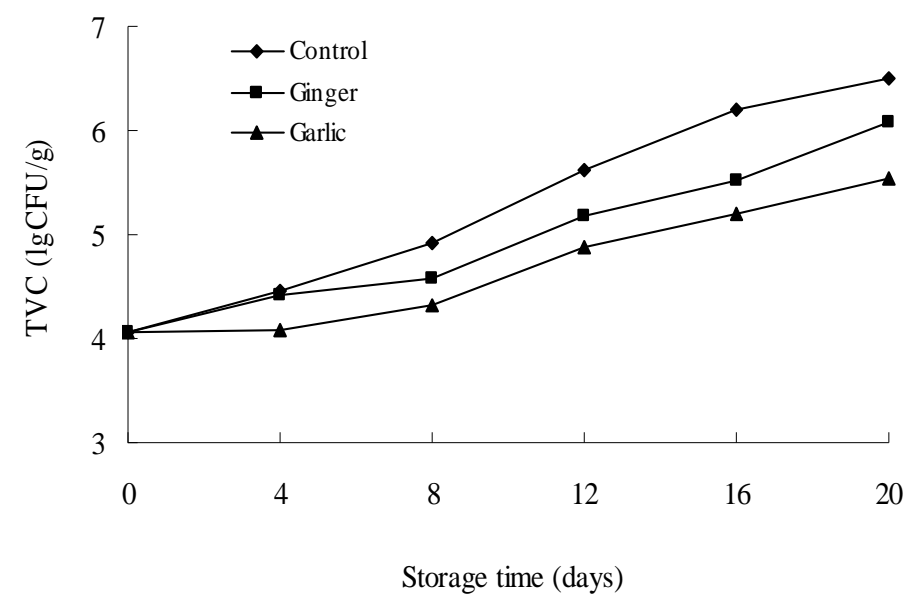

Fig. 1 Changes in TVC of turbot fillets during refrigerated storage

The spoilage of aquatic products is mainly caused by microbial action, and the TVC is an effective indicator for evaluating the quality of aquatic products [6]. As shown in Fig.1, with the extension of storage time, the TVC values of three treatment groups increased significantly. The 
TVC of control and ginger essential oil treatment samples exceeded $6 \mathrm{lg}$ CFU/g on days 16 and 20 of storage, which was considered as the upper acceptability limit for raw fish, while garlic essential oil coating samples didn't reach the value during the whole 20-day storage. The bacterial growth of control had no obvious stagnation stage over the entire storage. Samples of both ginger and garlic essential oil treatment group had significantly lower TVC number than that of control group during the storage of day 4 to $20(p<0.05)$, especially for the garlic essential oil group, which can more effectively control the growth of spoilage bacteria and prolong the shelf life of turbot fillets.

\subsection{Effects of essential oils coating on TVB-N of turbot fillets}

TVB-N has been widely used as an indicator for fish spoilage. Changes in TVB-N values of samples during refrigerated storage were presented in Fig.2. The initial TVB-N value of fresh turbot muscle was $4.5 \mathrm{mg} \mathrm{N} / 100 \mathrm{~g}$, which indicated that the turbot fillets were of good quality[7]. With the increasing storage time, the TVB-N values of all samples increased significantly $(p<0.05)$. TVB-N values increased slowly in the initial 4 days of storage and then showed a rapid growth trend. Lower TVB-N values $(p<0.05)$ were found in the essential oil treated samples compared with the control during the storage. The TVB-N value of control group reached $30.06 \mathrm{mg}$ N/100g on the 16th day, which exceeded limit of acceptability of $30 \mathrm{mg} / 100 \mathrm{~g}$ muscle. However, the TVB-N values of essential oil coating samples were still remained below the upper limit of acceptability till the storage ended. It was reported that garlic essential oil contains allicin, allyl sulfide and some other phenolic ingredients[8], which can inhibit the bacterial activity and enzyme activity in fish, reduce the protein degradation, and effectively controlled TVB-N value of turbot fillets during storage.

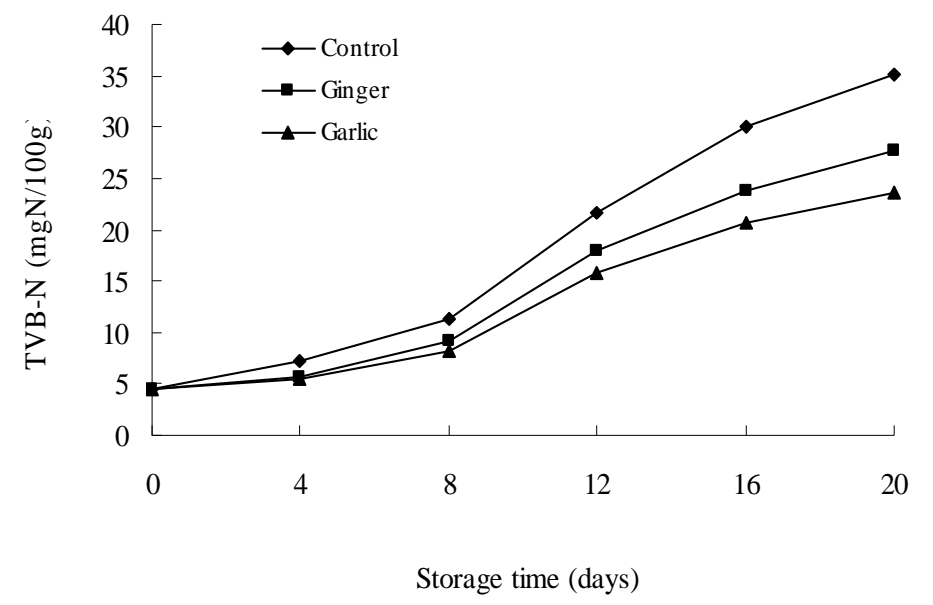

Fig. 2 Changes in TVB-N content of turbot fillets during refrigerated storage

\subsection{Effects of essential oils coating on $\mathrm{pH}$ of turbot fillets}

Changes in $\mathrm{pH}$ of turbot fillets during storage were shown in Fig.3. The initial $\mathrm{pH}$ of the fillets was about 7 . During storage, the $\mathrm{pH}$ values decreased in the initial 8 days of storage and then increased gradually from the 8 th day to the end. The initial decrease of $\mathrm{pH}$ value might be attributed to the lactic acid produced by muscle glycogen fermentation and the generation of phosphoric acid produced by the decomposition of ATP. Increasing in $\mathrm{pH}$ value with the storage time may be associated with the accumulation of alkaline substances, including ammonia and trimethylamine derived from fish spoilage microbial action. Similar observations were found by fan [9]. The samples reached the lowest $\mathrm{pH}$ values on the 8th day of storage. The $\mathrm{pH}$ values of both essential oil treated samples were consistently lower than that of the control during storage. Therefore, the lower $\mathrm{pH}$ value of essential oil treatment samples might inhibit the endogenous proteases activity, and 
maintained lower $\mathrm{pH}$ values.

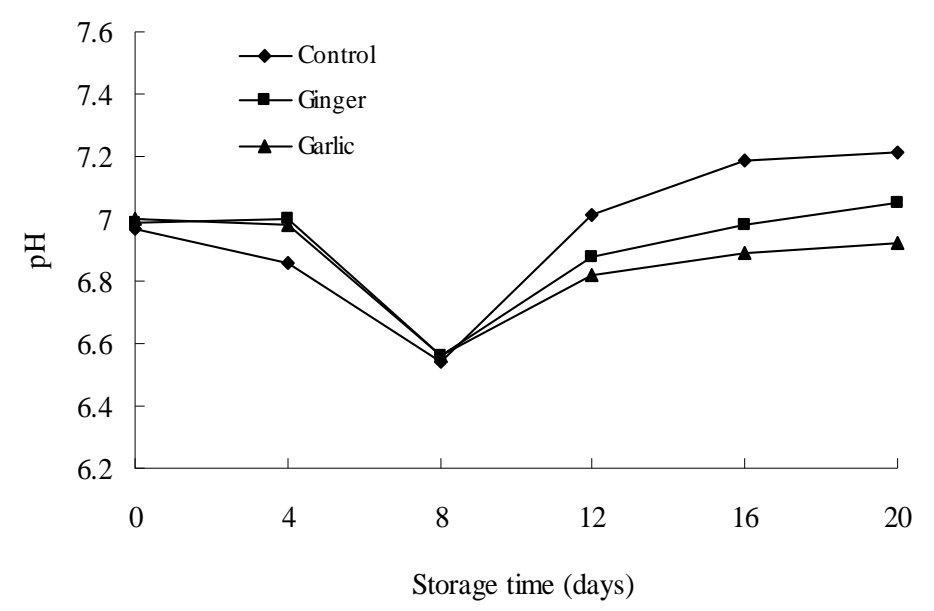

Fig. 3 Changes in $\mathrm{pH}$ of turbot fillets during refrigerated storage

\subsection{Effects of essential oils coating on texture profiles of turbot fillets}

Changes of hardness and chewiness for textural evaluation are shown in Table 1. The hardness and chewiness of turbot fillets decreased significantly as the storage time increased $(p<0.05)$. The control sample had the fastest softening rate, losing about $59.18 \%$ of its hardness over the whole storage of 20 days. The hardness of ginger and garlic essential oil coating samples also decreased but to a lesser extent, $42.89 \%$ and $52.71 \%$, respectively. Similar changes were also observed in chewiness parameters. Textural changes of turbot fillets during storage can be mainly due to the degradation of fish flesh by both the endogenous enzymes action and microbial activity[10]. Cai et al. [7] found that texture properties of turbot fillets with essential oil fumigation treatments decreased significantly during refrigeration, which was in agreement with our results. In the present study, texture properties could be delayed by essential oil treatments especially for garlic oil.

Table 1 Changes in hardness and chewiness of turbot fillets during refrigerated storage

\begin{tabular}{lcccccc}
\hline & \multicolumn{6}{c}{ Storage time (days) } \\
\cline { 2 - 7 } & \multicolumn{1}{c}{0} & 4 & 8 & 12 & 16 & 20 \\
\hline Hardness & & & & & & \\
Control & $2269.80 \pm 20.45$ & $1553.94 \pm 16.56$ & $1321.50 \pm 15.09$ & $994.60 \pm 13.98$ & $950.61 \pm 7.80$ & $926.64 \pm 8.04$ \\
Ginger & $2269.80 \pm 20.45$ & $1795.96 \pm 17.09$ & $1686.07 \pm 17.66$ & $1357.81 \pm 12.54$ & $1125.91 \pm 10.93$ & $1073.40 \pm 11.99$ \\
Garlic & $2269.80 \pm 20.45$ & $1895.89 \pm 15.00$ & $1787.38 \pm 13.09$ & $1452.81 \pm 14.27$ & $1353.07 \pm 14.32$ & $1296.24 \pm 13.51$ \\
Chewines & & & & & & \\
Control & $798.67 \pm 3.05$ & $636.44 \pm 8.16$ & $557.49 \pm 7.89$ & $520.07 \pm 5.73$ & $495.06 \pm 6.99$ & $494.42 \pm 6.16$ \\
Ginger & $798.67 \pm 3.05$ & $684.17 \pm 9.45$ & $646.84 \pm 8.88$ & $577.62 \pm 7.36$ & $526.85 \pm 6.71$ & $507.11 \pm 7.46$ \\
Garlic & $798.67 \pm 3.05$ & $722.86 \pm 9.14$ & $654.83 \pm 8.71$ & $625.33 \pm 8.35$ & $569.11 \pm 5.57$ & $541.78 \pm 6.18$ \\
\hline
\end{tabular}

\section{Conclusions}

The essential oil treated coatings had better effect than control in many indexes including the total number of colony, TVB-N content, $\mathrm{pH}$ and texture parameter. Results suggested that garlic essential oil treatment presented optimal effects in microbial growth inhibition, protein decomposition control, nucleotide breakdown passivation and sensory quality control. The essential oil coating treatment is a promising approach to maintain quality of aquatic products during storage. 


\section{Acknowledgements}

This work was funded by the National Natural Science Foundation of China (No. 31701631), Open Foundation of Food Resource Development and Quality Safety Key Lab of Jiangsu Province (SPKF201401*), and the China Postdoctoral Science Fouandation (No. 2016M602635).

\section{References}

[1] Özogul Y, Özogul F, Kuley E, et al. Biochemical, sensory and microbiological attributes of wild turbot (Scophthalmus maximus), from the Black Sea, during chilled storage. Food Chemistry. vol. 99(2006): p. 752-758.

[2] Tongnuanchan P, Benjakul S. Essential Oils: Extraction, Bioactivities, and Their Uses for Food Preservation. Journal of Food Science. vol. 79(2014): p. 1231-1249.

[3] Kim H S, Lee S H, Byun Y, et al. 6-Gingerol reduces Pseudomonas aeruginosa biofilm formation and virulence via quorum sensing inhibition. Scientific Reports. vol. 5(2015): p. 8656.

[4] Teixeira B, Marques A, Pires C, et al. Characterization of fish protein films incorporated with essential oils of clove, garlic and origanum: Physical, antioxidant and antibacterial properties. LWT-Food Science and Technology. vol. 59(2014): p. 533-539.

[5] FOSS. Determination of total volatile basic nitrogen of fresh fish and frozen fish. Application Sub Note. (2002): p. 16.

[6] Koutsoumanis K, Stamatiou A, Skandamis P, et al. Development of a microbial model for the combined effect of temperature and $\mathrm{pH}$ on spoilage of ground meat and validation of the model under dynamic temperature conditions. Applied and Environmental Microbiology. vol. 72(2006): p. 124-134.

[7] Cai L, Cao A, Li T, et al. Effect of the fumigating with essential oils on the microbiological characteristics and quality changes of refrigerated turbot (Scophthalmus maximus) fillets. Food and Bioprocess Technology. vol. 8(2015): p. 844-853.

[8] Rahman M S. Allicin and Other Functional Active Components in Garlic: Health Benefits and Bioavailability. Functional Foods. vol. 10(2007): p. 245-268.

[9] Fan W, Chi Y, Zhang S. The use of a tea polyphenol dip to extend the shelf life of silver carp (Hypophthalmicthys molitrix) during storage in ice. Food Chemistry. vol. 108(2008): p. 148-153.

[10] Li T, Li J, Hu W, et al. Shelf-life extension of crucian carp (Carassius auratus) using natural preservatives during chilled storage. Food Chemistry. vol. 135(2012): p. 140-145. 